\title{
Evaluating correlation of the native Inaba strain with the dominant isolated strains in outbreaks occurred in Iran at 2013 by Pulsed Field Gel Electrophoresis
}

\author{
Massoud Hajia' ${ }^{1}$ Alireza Dolatyar², Marjan Rahnami Farzami ${ }^{3}$, Mohsen Imani ${ }^{4}$, Roghieh Saburian ${ }^{4}$, \\ Mohamad Rahbar ${ }^{1}$ \\ ${ }^{1}$ Department of Molecular Biology, Research center of Health Reference Laboratories, Ministry of Health and Medical \\ Education, Tehran, Iran \\ ${ }^{2}$ Research center of Health Reference Laboratories, Ministry of Health and Medical Education, Tehran, Iran \\ ${ }^{3}$ Research center of Health Reference Laboratories, Ministry of Health and Medical Education, Tehran, Iran \\ ${ }^{4}$ Center for Communicable Diseases Control. Ministry of Health and Medical Education, Iran
}

\begin{abstract}
Objectives: The aim of this study was to analyze the isolated cholera strains at outbreak 2013 for studying the their similarity and compare their homology in order to find out the route of infection either emerge from abroad or reemerge from inside native strains.

Methods: All diagnosed $V$. cholerae isolates were entered to the study after re-identification at referral laboratory of Health Ministry based on standard procedures. These specimens were examined for specific serogroups by $\mathrm{O} 1$ polyvalent and Ogawa/Inaba nonspecific antisera and tested by MIC Test Strip Method against Ciprofloxacin, Nalidixic Acid, Cefixime, Ampicillin, Tetracycline, Trimethoprim-Sulfamethoxazole, and Erythromycin.

Results: A total of 257 clinical Vibrio cholerae was isolated in an outbreak of Iran at 2013. The dominant causative type was Inaba. In Antibiotic susceptibility test isolates were $100 \%$ resistant to all except Erythromycin that just $23 \%$ of strains were sensitive. Homology of isolates was investigated through genotyping by PFGE method and their clonality was compared with previous isolated Iranian native strain. Overall $92 \%$ of analyzed strains showed a homolog pattern. These strains were located in 8 clusters. Although isolated strains at 2011 had $80 \%$ homology with recent isolates, located in complete distinct cluster than all strains isolated at 2013. PFGE analysis revealed no dissimilarity between those stains resistant and sensitive to Erythromycin.
\end{abstract}

Conclusion: This study confirmed that isolated Inaba strains at 2013 had different clonality pattern in PFGE than previously identified, suggested have foreign route from the neighboring countries. J Microbiol Infect Dis 2016;6(4): 184-189

Key words: Vibrio cholera, Outbreak, Inaba, PFGE

\section{INTRODUCTION}

According to the World Health report in 2012, it is estimated that approximately 3-5 million cholera cases occur every year while only a small portion of these cases are reported to WHO especially in our region countries such as Afghanistan, Pakistan, India and Bangladesh [1]. Based on the analysis of World Health Organization (WHO) data, cholera outbreaks are explained by environmental and climatic factors especially some disaster such as flood and earthquake $[2,3]$.
Previous studies indicated the trend of cholera isolates was toward Inaba during 2005-2010 outbreaks [4-6]. But the cause of registered outbreaks that spread throughout the whole country was Ogawa serotype at 2011 [7]. Emergence of this serotype was an alarm in Iran after about seven years for import of new $V$. cholerae clones from out of the country. However all documents of the Center for Disease Control of Iran shown it switched to Inaba after two years at 2013. It could be re-emerging of native reservoir or imported strains from abroad outbreaks. Based on the previous study a few Inaba had been isolated from sporadic cases [7].

Correspondence: Massoud Hajia, Health Reference Laboratory of Iran, Ministry of Health and Medical Education, No.48, Shahrokh Alley, Zartoshtian St., Hafez Av., Tehran, Iran Email: massoudhajia@yahoo.com Received: 09 December 2015; Accepted: 27 September 2016 
Molecular typing techniques, such as RAPD, ribotyping and multilocous enzyme electrophoresis (MEE), have been employed to study genetic relatedness $[8,9]$. However PFGE has been able to provide a considerable role in epidemiological investigations because it has a high discriminatory power [11]. The PFGE protocol for cholera has been validated and standardized. It is accepted the technique to be able to discriminate the data of those participating laboratories and compare their correlations and homology. Therefore it can be applied as strong tools to monitoring and controlling of the enteric pathogen [12].

Pulse-field gel electrophoresis (PFGE) is considered as the "gold standard" molecular typing method for food borne pathogens, illustrating high discriminatory power for epidemiology investigations. This method is able to support epidemiological data in describing how a $\mathrm{V}$. cholerae 01 isolate can be emerge from abroad or reemerge from native strains. Based on the previous study seven pulsotypes was reported that three type were dominant throughout the country and for sporadic ones [7], while in those previous study just two types had been detected $[5,6]$. The objective of this study was analysis the isolated Inaba strains at outbreak 2013 to study the similarity of the isolated strains and compare their homology in order to find out the route of infection either emerge from abroad or reemerge from inside native strains.

\section{MATERIALS AND METHODS}

All patients suspected to have cholera were entered to this study. All $V$. cholerae isolates were diagnosed in any provinces at their local laboratories based on standard procedures [13,14]. The first five diagnosed V.cholerae strains were transferred to the Health Reference Laboratory from each province for re-identified as referral laboratory for final confirmation as established surveillance system by the Ministry of Health and Higher Education $[15,16]$. These specimens were examined for specific serogroups by $\mathrm{O} 1$ polyvalent and Ogawa/Inaba monospecific antisera (BD, Becton Dickinson Co. USA) after identifying use standard biochemical and bacteriological tests.

Antimicrobial susceptibility: Those confirmed V.cholerae isolates was tested by MIC Test Strip Method using Liofilchem (CE IVD approved, Italy) against Ciprofloxacin (CIP), Nalidixic Acid (NA), Cefixime (CFM), Ampicillin (AMP), Tetracycline (TE), Trimethoprim-Sulfamethoxazole (SXT), and
Erythromycin (E). Following organisms were used as quality control strains for MIC E-testing; E.coli (ATCC 25922), S. aureus (ATCC 29213), and P. aeruginosa (ATCC 27853) [17].

PFGE: Genotyping of isolates was performed by pulsed-field gel electrophoresis using Pulse Net standard procedure for $V$. cholera [12]. The whole agarose-embedded genomic DNA from $V$. cholera was prepared. The conditions used for separation were as follows: An isolated colony was streaked from test cultures to Trypticase Soy Agar with 5\% defibrinated sheep blood (TSA-SB) plates incubated overnight for confluent growth $[7,13]$.

Grown colonies within 14-18 hours were used to prepare cell suspension. Bacterial suspension was prepared in a cell suspension buffer $(100 \mathrm{mM}$ Tris:100 mM EDTA, pH 8.0) and adjusted to absorbance values of $0.8-1.0$ at a wavelength of $610 \mathrm{~nm}$ after which plugs were prepared with SeaKem Gold agarose (Lonza, Rockland, ME, USA) and proteinase $\mathrm{K}$. Bacterial plugs were lysed $(50 \mathrm{mM}$ Tris: 50 mM EDTA, pH $8.0+1 \%$ Sarcosyl, and $25 \mu$ l Proteinase $\mathrm{K} 20 \mathrm{mg} / \mathrm{ml}$ )and washed by pre-heated sterile ultrapure water and sterile TE buffer six times in a $54-55^{\circ} \mathrm{C}$ water bath. Each plug was digested with forty units of Not I restriction enzyme (Fermentas). DNA molecular weight size marker was prepared by Xba I digestion of Salmonella enterica serotype BraenderupH9812 plugs. PFGE was carried out with CHEF Mapper XA System (Bio-Rad) using program explained by Pulse Net.

Image analysis. The fingerprinting pattern in the PFGE gel was analyzed using the computer software package BioNumerics 6.6 (Applied Maths, Belgium). After background subtraction and gel normalization, the fingerprint patterns were subjected to typing on the basis of banding similarity and dissimilarity using the Dice similarity coefficient and clustering based on the unweighted-pair group method using average linkages (UPGMA), as recommended by the software manufacturer, and results are graphically represented as dendrograms.

\section{RESULTS}

Totally 257 cholera cases were recorded by the authorities throughout the country and just in 12 provinces during the outbreaks occurred in 2013. The Highest Cholera rate $(55.25 \%)$ were recorded at Baluchistan with 142 cases while other engaged provinces were Kerman, Tehran, Fars, Hormozgan, Qazvin, Qom, Alborz, Golestan, Esfahan, Khorasan Jonubi and Razavi with 73, 10, 8, 8, 5, 3, 2, 2, 2, 
1 , and 1 cases respectively. The mean age of the confirmed patients was $25.52 \pm 11$. The mortality rate was $1.95 \%$. Out of 257 cases, $45(17.5 \%)$ were Iranian while the 210 registered cased were afghan $(81.71 \%)$, and the rest were Pakistani travelers. The Ratio of male to female and patients was $89.1 \%$.

According to the issued instructions released by Center for Disease Control and Prevention, just 118 specimens were sent for confirmation and 104 out of them were confirmed as cholera cases including 3 Ogawa and 101 Inaba strains. These three Ogawa strains were not considered in this comparison because they had not an important role in the current outbreak.

Antibiotic susceptibility test revealed 100\% resistance for Inaba serotypes to Nalidixic acid, Tetracycline and SXT and while all of them were sensitive to Ciprofloxacin, Cefixime and Ampicillin. Susceptibility test showed only $23 \%$ were sensitive to Erythromycin that were isolated from both Afghan travelers and Iranian citizen, although all strains were showed intermediated pattern from the second month of outbreak. Isolated specimen at 2011 (30-90), had different pattern. It was sensitive to all above antibiotics except SXT (Table 1).

Table 1. Results of susceptibility test

\begin{tabular}{|c|c|c|c|}
\hline \multirow{2}{*}{ Antimicrobial Agent } & \multicolumn{2}{|c|}{ Sensitive Intermediate } & \multirow{2}{*}{$\begin{array}{c}\text { Resistant } \\
2013\end{array}$} \\
\hline & 2013 & 2013 & \\
\hline Ciprofloxacin & $100 \%$ & $0 \%$ & $0 \%$ \\
\hline Nalidixic Acid & $0 \%$ & $0 \%$ & $100 \%$ \\
\hline Cefixime & $100 \%$ & $0 \%$ & $0 \%$ \\
\hline Ampicillin & $100 \%$ & $0 \%$ & $0 \%$ \\
\hline Tetracycline & $0 \%$ & $0 \%$ & $100 \%$ \\
\hline $\mathrm{SXT}^{*}$ & $0 \%$ & $0 \%$ & $100 \%$ \\
\hline Erythromycin & $23 \%$ & $77 \%$ & $0 \%$ \\
\hline
\end{tabular}

The genomic DNA of the 31 selected strains plus one belonging to outbreak 2011 was digested by Not I restriction enzyme, creating 17 to 20 fragments, the sizes of which ranged from 20.5 to 668.9 $\mathrm{kb}$. Cluster analysis by dendrogram of the gel images separated the $V$. cholerae biotype strains into some major clusters although generally all analyzed strains at 2013 showed $92 \%$ homology. These strains were located in 8 clusters. Strains isolated at 2011 were also less than $80 \%$ homology and were located in complete distinct cluster than all strains isolated at 2013.
PFGE analysis revealed no correlation between the stains resistant and sensitive to Erythromycin (Figure1), although susceptible strains were seen at first two weeks of commencing outbreaks (Table2).

Table 2. Susceptibility Results to Erythromycin of isolated strains Isolated at this study

\begin{tabular}{|c|c|c|c|c|c|}
\hline & $\begin{array}{c}\text { Code } \\
\text { No. }\end{array}$ & Region & Nationality & $\begin{array}{l}\text { Date of } \\
\text { Receiving } \\
\text { specimens }\end{array}$ & $\begin{array}{l}\text { Sensitivity to } \\
\text { Erythromycin }\end{array}$ \\
\hline 1 & $7-92$ & Bandar Abbas & Afghan & 4 Sep & Intermediate \\
\hline 2 & $8-92$ & Iranshahr & Afghan & 8 Sep & Sensitive \\
\hline 3 & $11-92$ & Iranshahr & Afghan & 8 Sep & Intermediate \\
\hline 4 & $15-92$ & Iranshahr & Afghan & 8 Sep & Sensitive \\
\hline 5 & $19-92$ & Iranshahr & Afghan & 8 Sep & Intermediate \\
\hline 6 & $30-92$ & Zahedan & Afghan & 9 Sep & Intermediate \\
\hline 7 & $37-92$ & Zahedan & Afghan & 9 Sep & Intermediate \\
\hline 8 & $43-92$ & Qazvin & Afghan & 11 Sep & Sensitive \\
\hline 9 & $44-92$ & Qom & Iranian & 15 Sep & Intermediate \\
\hline 10 & $45-92$ & Karaj & Iranian & 16 Sep & Intermediate \\
\hline 11 & $47-92$ & Gorgan & Iranian & 16 Sep & Sensitive \\
\hline 12 & $48-92$ & Qazvin & Iranian & 17 Sep & Sensitive \\
\hline 13 & $49-92$ & Tehran & Afghan & 17 Sep & Sensitive \\
\hline 14 & $50-92$ & Tehran & Iranian & 18 Sep & Intermediate \\
\hline 15 & $51-92$ & Jiroft & Iranian & 19 Sep & Intermediate \\
\hline 16 & $54-92$ & Jiroft & Iranian & 19 Sep & Intermediate \\
\hline 17 & $57-92$ & $\begin{array}{l}\text { Rudbar-e- } \\
\text { Baluchistan }\end{array}$ & Afghan & 19 Sep & Intermediate \\
\hline 18 & $59-92$ & Tehran & Iranian & 23 Sep & Intermediate \\
\hline 19 & $60-92$ & Qazvin & Afghan & 24 Sep & Intermediate \\
\hline 20 & $63-92$ & Zahedan & Afghan & 24 Sep & Intermediate \\
\hline 21 & $67-92$ & Zahedan & Afghan & 24 Sep & Intermediate \\
\hline 22 & $78-92$ & Zahedan & Afghan & 24 Sep & Intermediate \\
\hline 23 & $87-92$ & Tehran-Ray & Iranian & 30 Sep & Intermediate \\
\hline 24 & $88-92$ & Karaj & Iranian & 30 Sep & Intermediate \\
\hline 25 & $89-92$ & Tehran & Iranian & 7 Oct & Intermediate \\
\hline 26 & $91-92$ & Bandar Abbas & Iranian & 7 Oct & Intermediate \\
\hline 27 & $94-92$ & Bandar Abbas & Afghan & 14 Oct & Intermediate \\
\hline 28 & $96-92$ & Kashan & Afghan & 15 Oct & Intermediate \\
\hline 29 & $97-92$ & Tehran-Ray & Afghan & 15 Oct & Intermediate \\
\hline 30 & $102-92$ & Larestan-Fars & Afghan & $3 \mathrm{Nov}$ & Intermediate \\
\hline 31 & $103-92$ & Bandar Abbas & Afghan & $12 \mathrm{Nov}$ & Intermediate \\
\hline
\end{tabular}




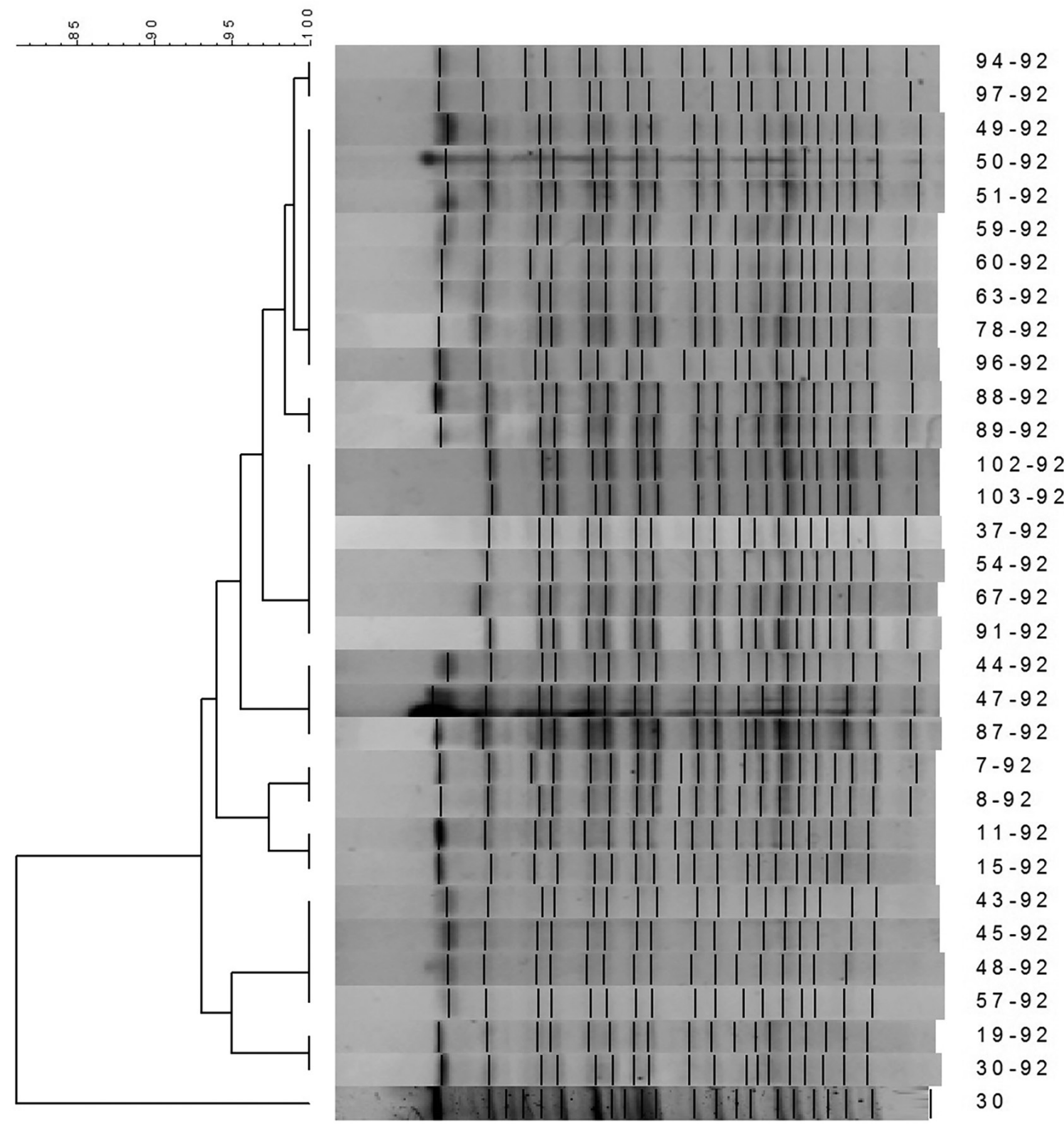

Figure1. Correlation PFGE analysis of Tested Specimens

\section{DISCUSSION}

Mafi and his colleagues have studied the cholera outbreaks in Iran since 2010 to 201[18]. They have gathered all the data and concluded that Ogawa strains have reduced from 100 at 2010 to $1.17 \%$ at 2013 and rate of Inaba strains have increased to $98.8 \%$ in 2013 instead. However, similar studies indicated the trend of isolates toward Inaba during 2005-2010 outbreaks [5].

Among total reported cases $83.65 \%$ of the all cases involved with cholera in Baluchistan and
Kerman provinces. The Afghan travelers had the majority contribution of the cholera engagement in this out break (81.71\%). Therefore, it is expected the infection to be transmitted from abroad by the travelers and spread to other provinces from these two province such as previously outbreaks $[18,19]$, but needs to be confirmed by a molecular typing method.

Isolated strains have been analyzed by the PFGE method using software package BioNumerics for the outbreak 2011 [7]. Analysis results of PFGE in this study revealed the cholera isolates 
were in different clusters. Ogawa serotype was a main type of the outbreak of the 2011. This serotype was distributed through the country with six different patterns, while a few Inaba strains were only isolated with differentiated patterns from Ogawa serotype [7]. At the present study the diversity of cholera strains at the outbreak 2013 was studied and the results were compared with the homology of detected Inaba strains at 2011. We concluded the previous native Inaba strain could not have a role in 2013 outbreak.

PFGE results showed no correlation between pattern of our pulsotypes and susceptibility testing results. This result was also reported by different researches $[20,21]$. The V.cholerae strains isolated in this study had nearly similar susceptibility pattern, except in Erythromycin. The specimens isolated at 2011 that located in separated cluster had different susceptibility pattern, although those Inaba isolated strains with different result in Erythromycin results had no distinct PFGE patterns. However, it seems this issue needs to be more investigated in separate researches. Therefore, we sagest susceptibility testing should be performed for all confirmed isolates during the outbreaks not just for the first identified strains. It means, we need to revise our previous released sampling procedure.

In recent years, new pathogenic variants of $V$. cholerae have emerged and spread throughout many Asian and African countries [2]. On the other hand emergence of multidrug resistant $V$. cholera isolates has been reported frequently [5] that is a major problem in developing Countries, specially Iran surrounded by several neighboring countries engaging each with cholera problem. The number of male cholera patients, their nationality and the mean age all obviously confirmed emerging cholera from the abroad. Regarding above mentioned point performing an active surveillance program at least during summer season for all passengers from Afghanistan and Pakistan is recommended.

Although the main aim of establishment of Pulse Net in eastern Mediterranean region is to help for early detection, investigation on bacterial causes of outbreaks could not be traced in members causing a gap. It is also needs to mention we could not have access to all specimens therefore this study has performed on the limited number of isolates. It is clear that using recommended standardized procedures for pulsed-field gel electrophoresis (PFGE) would help to compare obtained data from different countries.

\section{CONCLUSION}

This study proved isolated Inaba strains were emerged from the neighboring countries and with separate clonality pattern in PFGE with the native. The analysis results was able to support epidemiological data in describing how a $V$. cholerae may distributed through the country. This study was also underlined the contribution of new variant of cholera EI Tor that had some dissimilarity with the previously isolated strains at outbreak of 2011.

\section{Acknowledgments}

The study was supported by Health Reference Laboratory and Center for Disease Control and Prevention of Ministry of Health and education. We hereby thanks from all staff in local centers of Iranian provinces helped us to perform this study by sending the specimens.

Declaration of Conflicting Interests: The authors declare that they have no conflict of interest.

Financial Disclosure: No financial support was received.

\section{REFERENCES}

1. World Health Organization. Cholera (South-East Asia Region). Available at http://www.who.int/topics/cholera/about/en/index.html. 2012.

2. Mukhopadhyay AK, Takeda Y, Balakrish Nair G. Cholera outbreaks in the El Tor biotype era and the impact of the new El Tor variants. Curr Top Microbiol Immunol 2014; 379:17-47

3. Sack DA, Sack RB, Nair GB, Siddique AK. Cholera. Lancet 2004; 363 (9404):223-33

4. Emch M, Feldacker C, Islam MS, et al. Seasonality of cholera from 1974 to 2005: a review of global patterns. Int J Health Geogr 2008; 7:31

5. Rahim M, Kazi BM, Bile KM, Munir M. The impact of the disease early warning system in responding to natural disasters and conflict crises in Pakistan. East Mediterr Heal 2010; 16:S114-S121.

6. Bakhshi B, Pourshafie MR, Assessing clonality of Vibrio cholerae strains isolated during four consecutive years (20042007) in Iran. Scand J Infect Dis 2009; 41:256-262.

7. Aliabad NH, Bakhshi B, Pourshafie MR, Sharifnia A. Molecular diversity of CTX prophage inVibrio cholerae. Lett Appl Microbiol 2014; 55:27-32.

8. Bakhshi B, Pourshafie MR, Navabakbar F, Tavakoli A, Genomic organisation of the CTX element among toxigenic Vibrio cholerae isolates. Clin Microbiol Infect 2008; 14(6): 562-568.

9. Hajia M, Rahbar M, Farzami MR, et al. Assessing clonal correlation of epidemic Vibrio cholerae isolates during 2011 in 16 provinces of Iran. Curr Microbiol 2015; 70:408-14.

10. Bhowmick TS, Das M, Roy N, Sarkar BL. Phenotypic and molecular typing of Vibrio cholerae $\mathrm{O} 1$ and 0139 isolates from India. J Infect 2007; 54: 475-82. 
11. Leal NC, Sobreira M, Leal-Balbino TC, de Almeida AM, de Silva MJ, Mello DM. Evaluation of a RAPD-based typing scheme in molecular epidemiology study of Vibrio cholerae O1, Brazil J Appl Microbiol. 2004; 96(3):447-454.

12. Zhou HJ, Diao BW, Cui ZG, Pang B, Zhang LJ, Kan B, Comparison of automated ribotyping and PFGE for subtyping of Vibrio cholerae. Lett Appl Microbiol 2009; 48 (6):726-731.

13. Arakawa E, Murase T, Matsushita S, et al. Pulsed-field gel electrophoresis-based molecular comparison of Vibrio cholerae $\mathrm{O} 1$ isolates from domestic and imported cases of cholera in Japan. J Clin Microbiol. 2000; 38: 424-6.

14. Cooper KL, Luey CK, Bird M, Terajima J, Nair GB, Development and validation of a PulseNet standardized pulsed-field gel electrophoresis protocol for subtyping of Vibrio cholerae. Foodborne Pathog Dis. 2006; 3(1):51-8.

15. Mahon C, Manuselis G, Lehman D. Textbook of diagnostic microbiology. 3rd edn. Saunders, Philadelphia 2006.

16. Cheryl A, Allen A, Joy G. Laboratory Methods for the Diagnosis of Epidemic Dysentery and Cholera Centers for Disease Control and Prevention Atlanta 1999:115.

17. WHO/CDC CSP/EDC/99.8. Laboratory methods for the diagnosis of epidemic dysentery and cholera. Centers for disease control and prevension. Atlanta, Georgia. 1999:41-51.

18. WHO. Guidance on regulations for the Transport of Infectious Substances. Available from: http://www.who.int/csr/resources/publications/biosafety/WHO_CDS_EPR_ 2007.
19. Clinical and Laboratory Standard Institute. Performance standards for antimicrobial susceptibility testing: 16th informational supplement. CLSI document M100-S11, Wayne, PA 2011.

20. Standard Operating Procedure for PulseNet PFGE of Vibrio cholera and Vibrio parahaemolyticus. PNL06. 2013. http:// www.cdc.gov/pulsenet/PDF/vibrio_pfge_protocol-508c.pdf.

21. Mafi M, Hajia M, Goya MM. A five years study on the epidemiological approaches of Cholera in Iran. Casp J of inter Med. 2016; 7(3):162-167.

22. Hajia M, Rahbar M, Saburian R, Antimicrobial Resistance Patterns of isolated Vibrio cholera Strains during 2011 till 2013. Intern J of Enteric Path. 2016; 4(1): e31719.

23. Bakhshi B, Salimi-Khorashad A. Clonal Dissemination of a Single Vibrio cholera O1 Biotype El Tor Strain in Sistan Baluchestan Province of Iran During 2013. Curr Microbiol 2015;71(2):163-169.

24. Chomvarin C, Jumroenjit W, Wongboot W, et al. Molecular Analysis and antimicrobial resistant of $\mathrm{V}$. cholera $\mathrm{O} 1$ in northeasdtern of Tailand. Souhteast Asia J Trop Med Public Health. 2012; 43(6):1437-1446.

25. Sherestha UT, Adhikari N, Maharjan R,et al. Multidrug resistant Vibrio cholerae $\mathrm{O} 1$ from clinical and environmental samples in Kathmandu city. BMC Infect Dis. 2015; 15:1-7. 